\title{
[PARA]métricas do patrimônio arquitetônico pelotense
}

[PARA]metrics from Pelotas' architectural heritage

\author{
Tássia Borges de Vasconselos \\ Universidade de São Paulo, Brasil \\ Tassiav.arq@gmail.com
}

Adriane Borda Almeida da Silva

Universidade Federal de Pelotas, Brasil

adribord@gmail.com

\author{
Luísa Félix Dalla Vecchia \\ Universidade Federal de Pelotas, Brasil \\ luisafelixd@gmail.com
}

\begin{abstract}
This paper describes a teaching methodology created in a graduate course for insertion of parametric design techniques in patrimonial architecture. The teaching strategy was based on structured schemes available in Grasshopper plug-in for Rhinoceros software, in which the students can manipulate the variables dynamically like a game. The selection of patrimonial architecture elements encouraged the students to create compositions of isometric procedures, and transformations with concepts of symmetry, proportion and recursion.
\end{abstract}

Keywords: Parametric Design; Heritage; Game; teaching methodology

\section{Introdução}

Este trabalho relata uma trajetória de implementação do ensino de desenho paramétrico junto às disciplinas de Modelagem Geométrica I e II do curso de Especialização em Gráfica Digital, da Faculdade de Arquitetura e Urbanismo da Universidade Federal de Pelotas, a partir da turma de 2013. A utilização do desenho paramétrico faz parte de uma teoria de projeto descrita em Oxman (2008), a qual compreende que a utilização do ambiente digital não pode ser apenas a transposição do método tradicional de representação, como uma "prancheta eletrônica". Mas sim, que deve ser estabelecido um método de projeto propriamente digital, envolvendo uma mudança de paradigma, em que são utilizadas amplamente as potencialidades das técnicas digitais de parametrização. O desenho paramétrico está baseado no uso de parâmetros para exploração de formas geradas por meio de associação geométrica (OXMAN, 2006).

No âmbito das disciplinas referidas o estudo de modelagem geométrica é promovido paralelamente ao propósito de construção de conhecimento sobre a arquitetura de interesse patrimonial da cidade de Pelotas. Para isto, elementos desta arquitetura são analisados e utilizados como objetos de referência para os exercícios de representação. Estes exercícios promovem a identificação de estratégias compositivas empregadas junto às fachadas deste patrimônio e logo, propõem métodos digitais dinâmicos, buscando evidenciar a conveniência para desencadear processos criativos a partir deste referencial.

Desta maneira, o desenho paramétrico passou a ser inserido junto ao estudo de transformações geométricas, de técnicas de geração e controle da forma. As tecnologias digitais utilizadas para isto recorrem ao uso de linguagem de programação visual.
As turmas compõem um grupo heterogêneo de estudantes, constituído por profissionais de Artes, Design e Arquitetura e Urbanismo. Até então, todos eles, em seu processo formativo de graduação, não tinham tido contato com tal método projetual.

Por outro lado, o grupo de docentes que conduz esta Especialização a utiliza como laboratório para seu próprio processo formativo, tendo em vista a condição de desenvolvimento desta área estar atrelada à constante atualização sobre o estado da arte das tecnologias de representação gráfica digital. Esta pós-graduação foi criada em 1999, tendo inicialmente as práticas docentes centradas em técnicas de realidade virtual para a obtenção de visualizações estáticas, animadas e em tempo real (Brod et al., 2011). Logo, avançaram para as técnicas de realidade aumentada (Nunes et al., 2012) e, nos últimos três anos foram sendo utilizadas tecnologias de fabricação digital, através de experimentos com impressão 3D e corte a laser (Brum, V, et. al. 2014) (VECCHIA, L. F. D, et al. 2015). No entanto, a associação do desenho paramétrico às representações de objetos de interesse patrimonial, ainda não tinham sido exploradas neste contexto de ensino de pós-graduação.

Ao passar a esta exploração, este grupo docente tem tido a oportunidade de observar como os profissionais, projetistas e estudantes de pós-graduação, que de alguma maneira já delimitaram um método projetual particular, se dispõem ao aprendizado de uma nova maneira de representar e criar. Maneira, esta, que exige um grau de abstração mais avançado, frente aos meios tradicionais, por não operar diretamente na representação figurativa, mas utilizando-se do número e da lógica algorítmica.

Nesta direção, os exercícios de desenho paramétrico junto ao Curso tem inicialmente $o$ intuito de demonstrar o potencial 
deste método para otimizar o processo de projeto de arquitetura. Este tipo de processo exige flexibilidade para modificações do todo ou de suas partes. As técnicas de desenho paramétrico foram associadas aos programas gráficos computacionais, permitindo tanto a especificação como o controle de parâmetros e hierarquias que correspondam a uma forma flexível às variações paramétricas (Burry, 2010).

Em contextos brasileiros estas técnicas vem sendo reconhecidas recentemente, como registrado em Flório (2011), existindo pouco tempo para já constituir uma geração de arquitetos que se utilizem efetivamente delas como método de projeto. Por ser um método que exige a abstração matemática formal, o currículo, no contexto em que se insere este estudo, não está preparado para absorver naturalmente este tipo de lógica projetual. Tem-se investido na utilização do desenho paramétrico associado à linguagem de programação visual, por meio do Software Rhinoceros juntamente com o plugin Grasshopper. Assim as relações entre os objetos são explicitamente descritas, por meio de conexões entre parâmetros e operações formais, estabelecendo interdependências entre os vários objetos. Sem, contudo, exigir do usuário a compreensão de uma linguagem de programação textual, não presente em sua formação.

Oxman (2008) conceituou o Digital Architectural Design (DAD), que caracteriza o projeto arquitetônico digital, o qual amplia as possibilidades criativas dirigidas ao projeto devido às abordagens digitais. Coutinho et al (2012) demonstram a potencialidade de trazer a abordagem de desenho paramétrico para o estudo de processos compositivos de arquitetura. Configuram primitivas de colunas, de acordo com o tratado de Alberti. Em tal experiência, o conceito de Arquitetura Paramétrica, referido em Canuto e Amorim (2010), fica evidenciado, tendo em vista a tradução de um processo projetual por meio de uma geometria associativa.
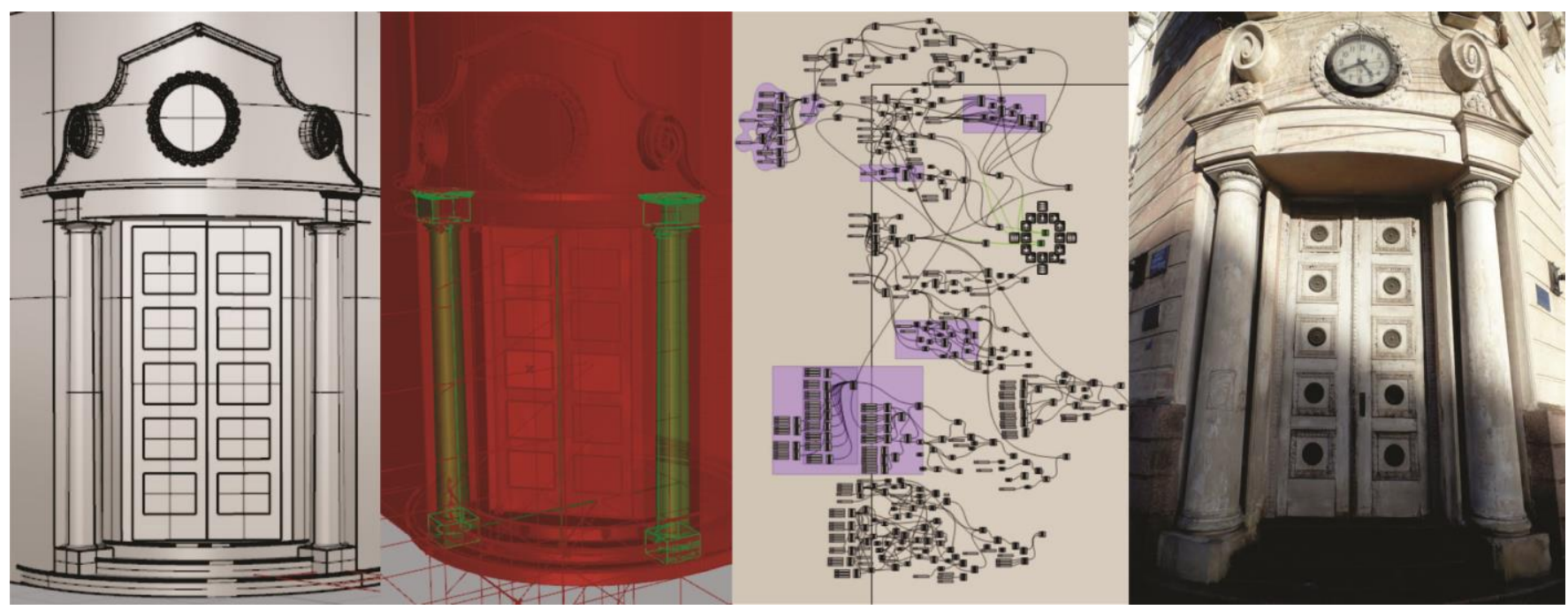

Cabe ainda salientar que nas primeiras experiências de inserção do desenho paramétrico no contexto do Curso de Especialização em questão, os estudantes foram incentivados ao uso, porém poderiam escolher, para o desenvolvimento da atividade final de representação, dentre os tipos de modelagem abordados nas disciplinas. Naquela ocasião, apenas uma estudante se encorajou a desenvolver esta atividade a partir do desenho paramétrico. A Figura 1 ilustra o resultado, desenvolvido pela primeira autora enquanto estudante deste curso. E, ainda deve-se destacar que esta estudante participou de processo formativo prévio de uso das ferramentas envolvidas. Frente à compreensão das dificuldades enfrentadas junto ao perfil específico do grupo de estudantes desta pós-graduação, buscaram-se alternativas. Paralelamente, estas mesmas autoras vinham desenvolvendo o mesmo movimento de inserção de desenho paramétrico junto aos contextos de graduação, como registrado em Vasconselos, Borda e Vecchia, 2014. Experiência que se desenvolve a partir da estratégia de jogos. E que tem se mostrado eficiente para introduzir as ferramentas de desenho paramétrico, por meio da manipulação dos esquemas de programação visual, a partir de estudos de geometria e representação junto aos estudantes de arquitetura de primeiro ano de formação.

\section{Metodologia}

O estudo foi caracterizado a partir das seguintes etapas: Revisão bibliográfica; Desenvolvimento de materiais didáticos; Experimentação e Análise e Sistematização dos resultados.

\section{Revisão bibliográfica;}

Partiu-se do repertório que as disciplinas de Modelagem Geométrica I e II buscam abarcar, utilizando-se da compreensão sobre procedimentos compositivos de arquitetura, especialmente àqueles gerais apresentados em Clarck \& Pause (1997) e Ching (1998); de simetria referenciados em Celani (2003); de proporção, reunidos em Doczi (1990) e de Recursão em Bovill (1996). das Finanças do Município, Pelotas/RS.

Fonte: Autoras, 2016. 
O estudo esteve apoiado também em Santos (2012) e Vaz e Celani (2012), trabalhos que trazem uma abordagem didática em contexto de ensino de geometria associado à utilização do desenho paramétrico por meio de programação visual.

\section{Desenvolvimento dos materiais didáticos;}

Foram construídas programações visuais que explorassem os conceitos sobre processos compositivos, utilizando-se fundamentalmente de parâmetros associados ao controle de relações proporcionais entre as partes dos elementos representados e de operações de simetrias e recursões.

Houve o propósito então de estabelecer uma dinâmica de jogo, buscando ambientar os estudantes com as técnicas de programação visual. Como estratégia, foi desenvolvido um esquema, ilustrado pela figura 2 , que exemplifica inicialmente a parametrização de um determinado elemento. No caso em questão tem-se uma curva diretriz e um perfil que por extrusão sobre esta curva gera a forma de um elemento qualquer de fachada. As imagens da linha superior desta mesma figura referem-se aos componentes que permitem controlar as proporções dos elementos, as quais constituem as métricas da arquitetura representada. $O$ esquema de parametrização do elemento iniciador está para ser totalmente manipulado e testado para observar a potencialidade ou não de gerar famílias de formas que possam abarcar os elementos a serem representados. Existe a mesma proposta para o uso dos esquemas das proporções, disponibilizados para serem associados a qualquer tipo de elemento.

\section{Experimentação:}

A experimentação, do processo de inserção de desenho paramétrico, ocorreu na disciplina de Modelagem Geométrica I (68 horas/aula) e II (34 horas/aula), com 7 alunos regulares, utilizando-se da metade da carga horária das disciplinas para os conteúdos teóricos, e um quarto para as atividades propriamente com a ferramenta em questão. É importante destacar que além da apresentação dos conteúdos através da programação visual são mantidas, paralelamente, as atividades com outros métodos de modelagem, a partir de diversos programas como: Sketchup, 3D MAX e o próprio Rhinoceros sem o apoio do plugin grasshopper. Caracterizase o propósito em evidenciar conceitos e potencialidades próprias de cada método e de cada ferramenta.

\section{Análise e Sistematização dos resultados;}

Nesta etapa, realizam-se as análises comparativas registrando-se as percepções docentes que diferenciam as experimentações didáticas de 2013 e 2014.

\section{Desenvolvimento do experimento}

A experimentação, em 2014, foi realizada com um grupo de maioria de Arquitetos e Urbanista (5 e 2 graduados em Artes), sendo que apenas uma estudante já havia tido um contato prévio com as ferramentas a serem utilizadas.
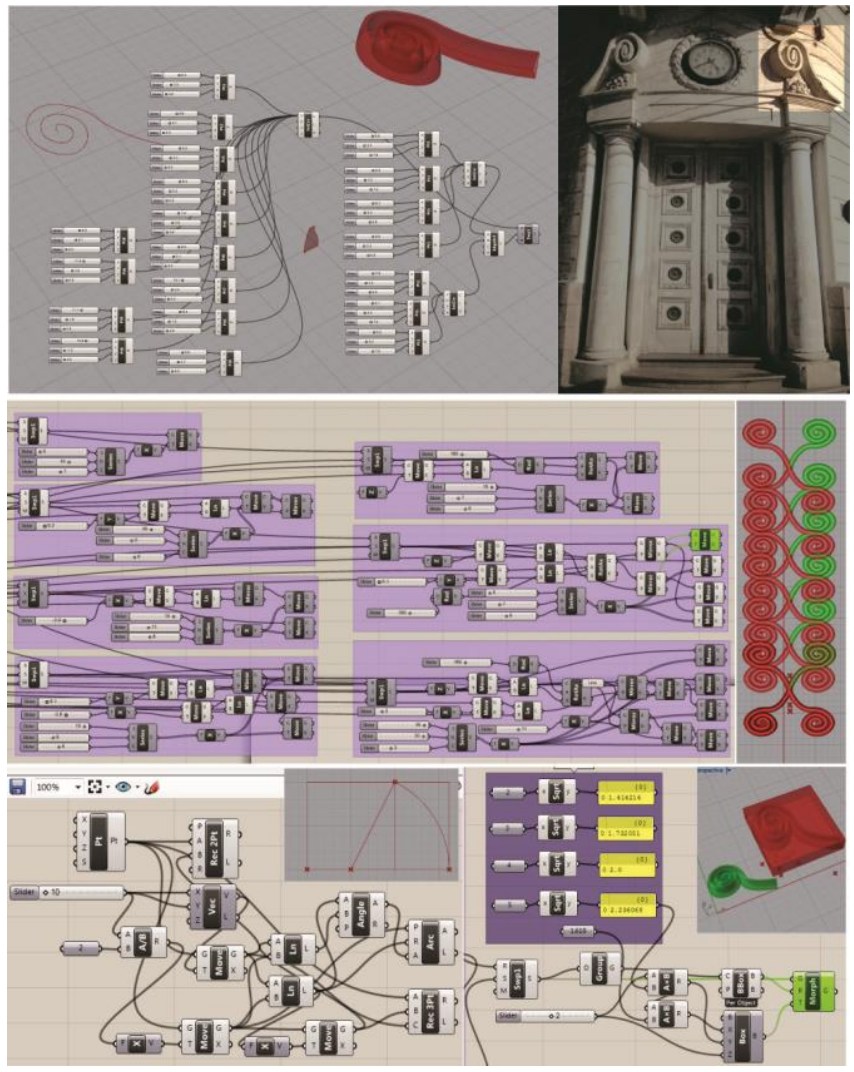

Figura 2: Na composição superior é apresentado o elemento que será utilizado, à esquerda em programação visual e à direita a foto com o elemento em destaque. Na composição central é apresentada as programações visuais sobre o conceito de simetria de frisos. $\mathrm{Na}$ composição superior é apresentada as programações visuais sobre o conceito de proporção .

Fonte autoras, 2016

\section{A. Compreensão dos conceitos a partir da programação visual, apresentação dos "Jogos compositivos":}

Conforme destacado anteriormente, para a compreensão das potencialidades da ferramenta, foram disponibilizadas as programações visuais anteriormente referidas. Estas foram apresentadas então como um jogo, promovendo a manipulação dos parâmetros para a geração de diferentes composições. Desta maneira, com a alteração nos sliders, cada um dos parâmetros variam dentro dos intervalos de valores previamente definidos, como ilustrado na Figura 3, onde visualiza-se em detalhe esta alteração. Um dos "jogos compositivos" desenvolvido exercita o conceito de simetria de friso, envolvendo essencialmente o movimento de translação. Os três parâmetros alteráveis foram estabelecidos como: $\mathbf{S}=$ distância do elemento iniciador do objeto existente, $\mathrm{N}=$ Distância entre os elementos, $\mathrm{C}=$ Quantidade de elementos. Assim, ao manipular estes parâmetros os estudantes obtinham composições a partir de variações formais. Também destaca-se na parte inferior da figura 3 os resultados formais explorados de outro jogo, explorando o conceito de simetrias 
cíclicas. Envolvendo também transformação por recursão e proporção.

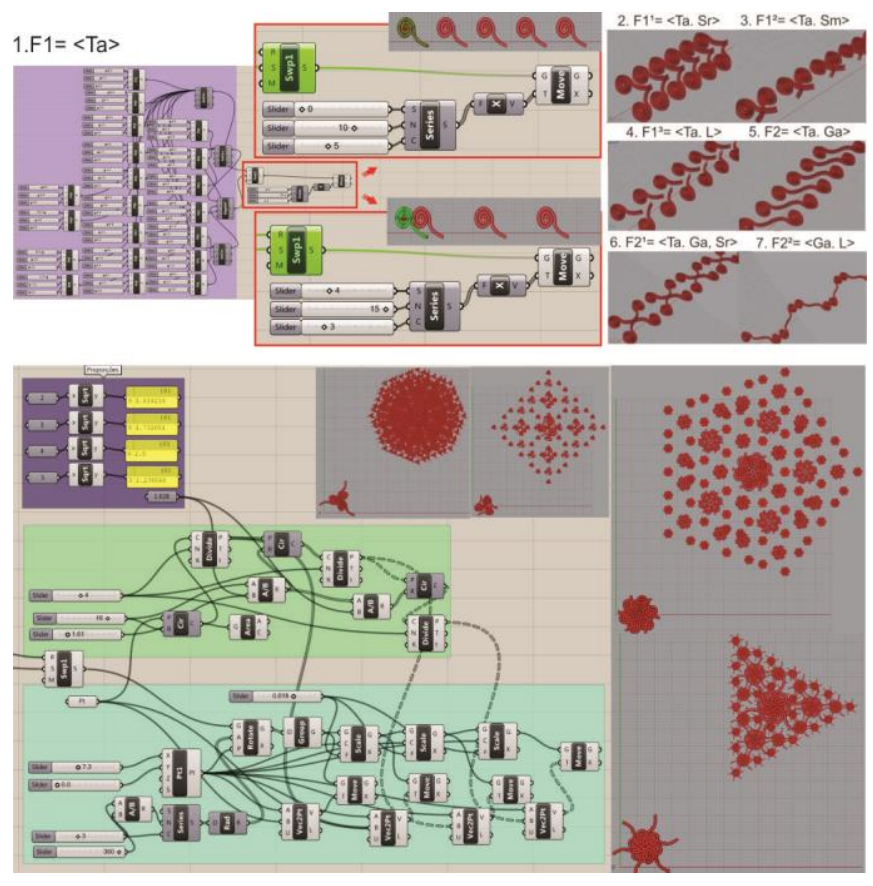

Figura 2: As Esquemas superiores relativos à geração de simetrias de frisos. Os esquemas inferiores às simetrias cíclicas, associando os conceitos de recursão e proporção.

Fonte autoras, 2016.

\section{B. Seleção e Modelagem de um elemento arquitetônico}

Nesta atividade, repetindo as edições anteriores da disciplina, os estudantes foram responsáveis por escolher um elemento do patrimônio arquitetônico da cidade, para 0 desenvolvimento dos exercícios de modelagem. Apenas uma das alunas optou pelo desenvolvimento de um elemento arquitetônico que não fazia parte deste escopo, porém como o elemento em questão continha uma complexidade formal apropriadas para as atividades a decisão da estudante foi apoiada. $O$ processo de modelagem também seguiu não sendo imposto, os estudantes adotaram ferramentas que se sentissem mais confortáveis/ motivados. Os exercícios ainda foram simplificados, adotando uma sequência didática de representação dos entes geométricos fundamentais: ponto, linha e plano, ilustrada na Figura 4. Na imagem da esquerda superior, os pontos, seguido das linhas e dos planos (imagem inferior da direita). Também foi exercitado o uso de componentes de programação visual como a primitiva "box" ou cubo, disponibilizada pelo grasshopper (imagem inferior da direita). Desta maneira, buscou-se explicitar os diferentes níveis de controle de uma mesma ideia de figura geométrica.

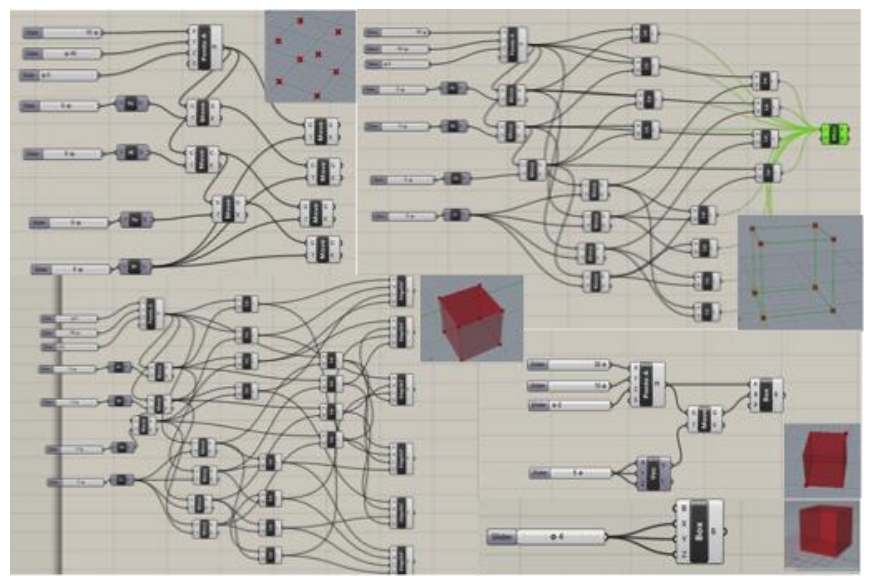

Figura 4: Programação visual da estratégia de reconhecimento do processo de modelagem.

Fonte autoras, 2016.

Posteriormente realizaram-se diferentes atividades como, por exemplo, sobre o conceito de superfícies poliédricas, quádricas e curvas. De maneira a ampliar o repertorio de estratégias de modelagem, por meio do desenho paramétrico, dos diferentes objetos. A imagem da Figura 5 apresenta um dos modelos desenvolvidos, neste caso da Caixa d'água de Pelotas. Entretanto, modelada por meio do software Auto Cad. Paralelamente as experimentações e demonstrando que mesmo observando as potencialidades do método 0 estudante não se sentiu encorajado de buscar a estruturação de um modelo associativo, o qual exige um esforço inicial de compreensão de sua lógica para ser programado, abstraído para que sua forma seja uma consequência, tal como. Coutinho et al (2012) demonstrou com as abstrações sobre as colunas de Alberti.

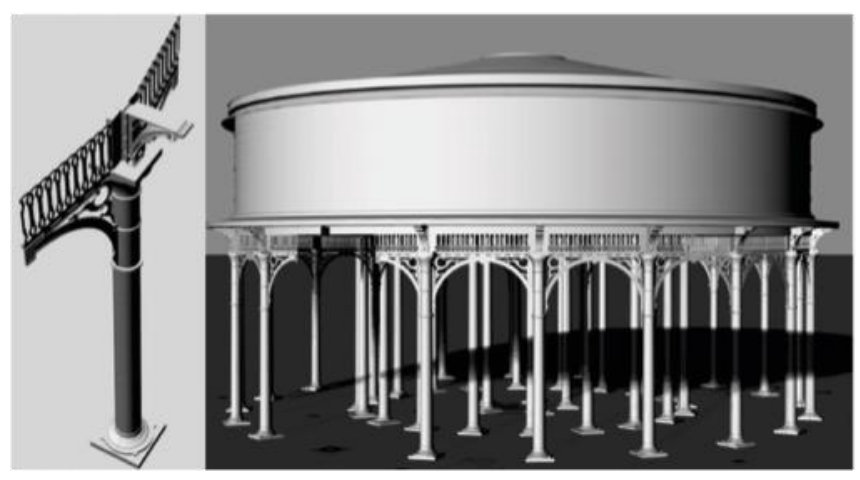

Figura 5: Modelo construído durante a atividade desenvolvida.

Fonte autoras, 2016.

\section{O uso dos "Jogos compositivos"}

Nesta outra atividade, os estudantes passaram a estudar os processos compositivos: proporção, simetria e recursão com o próprio elemento que optaram por modelar, ou ainda com o elemento em processo de desenvolvimento. Foram provocados a realizar modificações nas programações previamente estabelecidas. Os resultados diversos foram despertando o interesse e motivando o grupo para a produção 
de painéis para uma exposição capaz de compartilhar as descobertas deste potencial criativo do método. A Figura 6 exemplifica um dos trabalhos desenvolvidos mostrando, a esquerda, a obra de onde foi extraído o elemento de referência para a modelagem. Ao centro da figura, recortes dos esquemas, de programação visual, utilizados e, à direita, um dos painéis gerado. Neste caso, a estudante também não se sentiu encorajada de parametrizar o elemento em si, mas apenas as regras compositivas, valendo-se então do sistema de jogo que permite substituir 0 elemento base da composição.

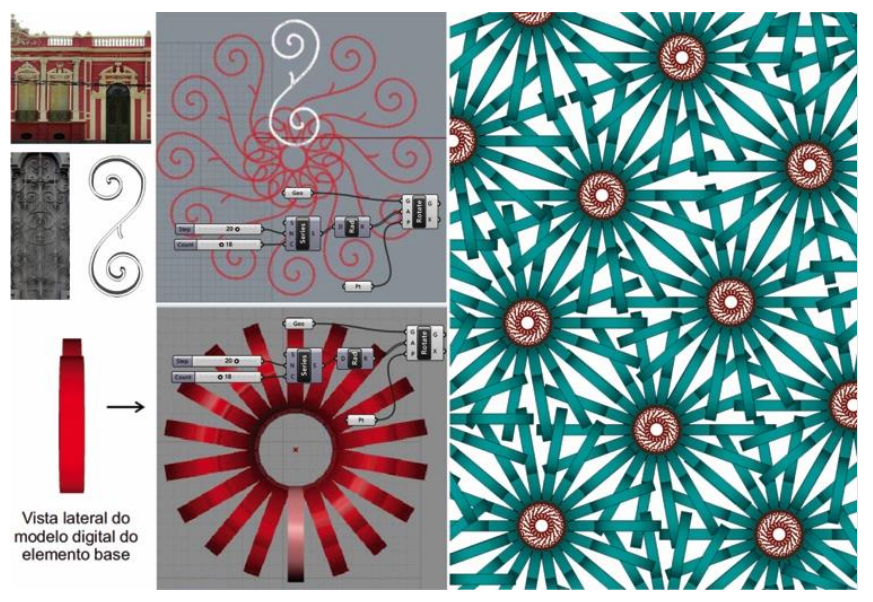

Figura 6: Programação visual das composições, e processo de exploração estabelecido por uma estudante.

Fonte autoras, 2016.

\section{A Exposição e a construção de uma leitura:}

A exposição dos painéis compositivos foi realizada durante a comemoração do dia Mundial do Turismo, no hall do Paço Municipal, exposição intitulada "[PARA]MÉTRICAS DO PATRIMÔNIO". O evento foi apoiado pela Secretaria de Desenvolvimento Econômico e Turismo, da cidade de Pelotas. Cada estudante selecionou dois ou três painéis dentre muitas das suas produções junto ao exercício., alguns destes apresentados na Figura 7. Junto a cada painel foram dispostos os materiais complementares, indicando onde 0 elemento arquitetônico representado se encontra na cidade, o processo de modelagem do elemento e o processo de criação da composição dos painéis. A partir da modelagem geométrica do elemento foi possível a impressão tridimensional do modelo, o que também fez parte do material complementar, como pode ser ilustrado com a imagem da direita na Figura 7.

Ainda, durante o processo de organização da exposição foi possível contar com a produção de uma leitura sobre este exercício, realizada pela Arquiteta Renata Requião, Professora da pós-graduação em Artes Visuais, se manifestou de forma textual sobre a exposição, destacando-se o trecho a seguir:

"A partir de esqueminhas que parecem conexões de eletricidade, ou conexões neuronais, fazem desenhos que são a um só tempo realidade e virtualidade, a um só tempo mundo real e mundo geometrizado. A base para esses desenhos vem dos elementos em ferro, reais, existentes em alguns lugares de nossa cidade. Neles vemos o que não vemos em nossa correria diária: elementos da Caixa d'água em ferro, na praça Piratinino de Almeida, uma mão francesa do Theatro Guarani, detalhes das grades nos peitoris de um casarão recuperado, numa esquina da cidade. Estes jovens, cujo nome se lê nesta exposição, não desenham nem modelam... estabelecem regras matemáticas, em geometria e lógica, capazes de gerar (como só um caleidoscópio, um fractal, uma folha ou uma cidade), tais composições, através de repetições, deformações, simetrias, e algumas recursões - figurações de sua intimidade com a cidade de Pelotas. Seremos mais felizes vendo daqui a cidade que eles viram." (Requião, 2014)


Figura 7: $\mathrm{Na}$ esquerda alguns painéis desenvolvidos, na direita os materiais complementares disponibilizados na exposição.

Fonte: Autoras, 2016. 


\section{Resultados e discussão}

Inicialmente tem-se como resultado a implementação do desenho paramétrico efetivamente no ensino das disciplinas em questão. A aplicação do método, até o presente momento em duas turmas do curso, refere-se, por um lado, à aceleração do processo de ensino/aprendizagem, tendo em vista que através dos momentos lúdicos de manipulação dos parâmetros os estudantes têm entendido a lógica de cada componente.

Durante o desenvolvimento da proposta estabelecida, no ano de 2014 na disciplina, 57\% dos alunos trabalharam com a exploração dos processos compositivos através de programação visual.

Mesmo com o aumento da utilização da programação visual, se comparado ao uso em 2013, a turma de 2014 continuou com apenas uma estudante desenvolvendo um modelo totalmente parametrizado por meio das técnicas apresentadas. Porém, a estudante de 2014 não tinha nenhuma experiência previa quanto ao uso dessas ferramentas. A sua experiência foi registrada em Rodrigues et al, (2014).

Além desta estudante, que desenvolveu o seu elemento utilizando a programação visual, dois outros estudantes se sentiram capazes de desenvolver algumas programações visuais, na etapa de exploração das composições, com o auxílio do grupo docente.

A iniciativa de associar, neste contexto, a utilização de elementos do patrimônio arquitetônico da cidade para gerar as composições atribuiu um diferencial para a experiência didática, os estudantes produziram um conjunto de composições que configurou uma exposição aberta à comunidade. A qualidade visual das composições geradas destacou o potencial criativo associado à agilidade e controle do processo a qual foi reconhecida fora do âmbito acadêmico através do evento cultural referido (exposição).

\section{Considerações Finais}

Considera-se que o trabalho alcançou os objetivos iniciais de inserção dos conhecimentos de desenho paramétrico associados ao patrimônio histórico para reconhecer processos diferenciados de geração formal no curso de pós-graduação. Ainda, por meio da exposição realizada, reafirma-se a postura do grupo de pesquisa, de valorização do patrimônio arquitetônico da cidade.

Destaca-se ainda uma possibilidade de maior difusão destas técnicas nas faculdades de Arquitetura e Urbanismo, contexto em que que ainda não são utilizadas amplamente na graduação. Acredita-se que os processos compositivos gerados podem servir como objeto de aprendizagem para as disciplinas de geometria da graduação.

A estratégia de jogo, exitosa apresenta-se também apropriada para profissionais que já tinham seus métodos estabelecidos.
Este processo ficou evidenciado a partir da surpresa dos alunos quanto as possibilidades e agilidade propiciada pelo desenho paramétrico.

Diferentes e numerosas soluções impensadas foram exploradas, diferentemente de quando estabelecidos através de recursos digitais que apenas traspõe o processo de representação tradicional para o ambiente digital. Assim, acredita-se que a experiência vai ao encontro do discurso estabelecido em Oxman (2008).

Ainda como seguimento a esta experiência, a prática está sendo revisada para aplicação em contexto de estágios iniciais de graduação em arquitetura e urbanismo, também associando as questões patrimoniais ao desenho paramétrico. Pretende-se assim explorar, uma maior apropriação desta prática, em contextos onde os estudantes ainda não têm um método de projeto estabelecido.

Entretanto, ainda cabe destacar que as expectativas eram maiores do que aquelas efetivamente vivenciadas. Os profissionais, projetistas e estudantes de pós-graduação envolvidos, que de alguma maneira já haviam delimitado um método projetual particular, pouco se dispuseram ao aprendizado de uma nova maneira de representar e criar. Maneira, esta, que exige um grau de abstração mais avançado. É possível que investindo desde os estágios iniciais de formação possam ter resultados mais animadores.

\section{Agradecimentos}

Agradecemos ao grupo de alunos da Especialização em Gráfica Digital pelo comprometimento com a experiência didática.

\section{Referências}

BOVILL, C. (1996). Fractal geometry in architecture and design. Boston: Birkhauser.

BROD, G. ; BORDA, A.B.A.S. (2011) Anamorfose na praça: um encontro do real e do virtual. In: XV SIGRADI - CONGRESSO DA SOCIEDADE IBERO-AMERICANA DE GRÁFICA DIGITAL, 2011, Santa Fé. Cultura Aumentada. Santa Fé - Argentina: FADU - UNL, 2011. v. 1. p. 130-133.

BURRY, Jane R. (2008) Mindful Spaces: Computational Geometry and the Conceptual Spaces in which Designers Operate. International Journal of Architectural Computing.

BRUM, V. ; VECCHIA, L. F. D. ; PEDERZOLLI, L. ; BORDA,A.B.A.S . (2015) Projeto e execução de modelagem para fabricação digital: aplicação em representação de patrimônio arquitetônico. In: Geometrias \& Graphica, Lisboa. ACTAS DA CONFERÊNCIA GEOMETRIAS \& GRAPHICA 2015. Lisboa: Universidade Lusíada de Lisboa, 2015. v. 1. p. 1-12

CHING, F.(1998) Arquitetura: Forma, espaço e Ordem. São Paulo: Martins Fontes.

CLARCK, R. H.; PAUSE, M. (1997) Arquitectura: temas de composición. Barcelona: Gustavo Gili, 1997.

CANUTO, R.; AMORIM, L. M. E.. (2010) Da arquitetura paramétrica ao urbanismo paramétrico. In: XIV Congreso de la Sociedad Iberoamericana de Gráfica Digital (SIGRADI). Disrupción, 
SIGraDi 2016, XX Congreso de la Sociedad Ibero-americana de Gráfica Digital 9-11, November, 2016 - Buenos Aires, Argentina

modelación y construcción: diálogos cambiantes. Bogotá: Ediciones Uniandes,. p. 419-422

DOCZI, G.. (1990) O poder dos limites: harmonias proporções na natureza. São Paulo: Mercuryo, 150 p.

FLORIO, W. (2009). Modelagem Paramétrica no Processo de Projeto em Arquitetura. In: SBPQ 2009, São Carlos: USP, p. 571-582.

GODOI, G., CELANI, G. (2012) "Alberti Digital - Estudo da Influência do Tratado Albertiano na Arquitetura Colonial Brasileira. In: XVI SIGRADI - Congresso da Sociedade Ibero Americana de Gráfica Digital. Forma (in)formação. Fortaleza: Expressão Gráfica e Editora, v.01. p.611-614, 2012.

NUNES, C. S.; SCHNEID, G.; VASCONSELOS, T. B ; SILVEIRA, A. ; BORDA, A. S. .(2012) O estudo de igrejas barrocas de ouro preto através de tecnologias avançadas de representação e visualização. In: Iv Congreso Internacional De Expresión Gráfica En Ingeniería, Arquitectura y carreras afines, La Plata. Gráfica del diseño: Tradición e innovaciones. Cordoba: Facultad de Arquitectura, Urbanismo y Diseño, p. 285-290.

OXMAN, Rivka.(2006) Theory and design in the first digital age. Design Studies. Londres: Elsevier. Disponível em: <http://www.technion.ac.il/>. Acesso em: 12/02/2015

OXMAN, R. "Digital architecture as a challenge for design pedagogy: theory, knowledge, models and medium". Design Studies. Great Britains: Elsevier, v 29 n 2 p.99-120, 2008.

SANTOS, A. C., VAZ, C. E. V., PEREIRA, D. C., DUARTE, M. E. R., PINHEIRO, J. V.L. (2012). O uso das ferramentas de desenho paramétrico no ensino de geometria gráfica - O caso dos poliedros. In: Iv Congreso Internacional De Expresión Gráfica En Ingeniería, Arquitectura y carreras afines, La Plata. Gráfica del diseño: Tradición e innovaciones, Cordoba: Facultad de Arquitectura, Urbanismo y Diseño, p. 291- 294.

REQUIÃO, R. (2014). Coisas do olhar: magia visual nas formas do ferro. In: Catálogo [PARA]métricas do patrimônio Pelotense, p. 2 -5 .

RODRIGUES, M.; VECCHIA, L. F. D. ; VASCONSELOS, T. B. ; PIRES, J. F. ; BORDA, A. S. . Referenciais do passado e representações do futuro: um exercício didático com os painéis de Erwin Hauer. In: EGRAFIA, 2014, Rosario. Revisiones del futuro Previsiones del pasado. Rosario, 2014.

VECCHIA, L. F. D. ; BORDA, A.B.A.S ; VEIGA, M. VASCONSELOS, T. B; BORGES, L. F. . Tactile models of elements of architectural heritage From the building scale to the detail. In: 16th International Conference CAAD Futures 2015, 2015, São Paulo. The next city - New technologies and the future of the built environment. Campinas: Universidade Estadual de Campinas, 2015. v. 1. p. 434-446.

VASCONSELOS, T. B.; BORDA, A. S. ; VECCHIA, L. F. D. .( 2014) A parametrização como experiência prévia para estruturação de métodos projetuais em arquitetura. In: SIGRaDi - Congreso de la Sociedad Iberoamericana de Gráfica Digital, 2014, Montevideo. Design in freedom. Montevideo: Manuel Carballa,. p. 297-301. 